\title{
Direct Oral Anticoagulants and Interstitial Lung Disease: Emerging Clues from Pharmacovigilance
}

\author{
Emanuel Raschi ${ }^{1}$ (i) $\cdot$ Michele Fusaroli $^{1} \cdot$ Igor Diemberger $^{2} \cdot$ Elisabetta Poluzzi $^{1}$
}

Published online: 26 August 2020

C) Springer Nature Switzerland AG 2020

\section{Dear Editor,}

Evidence has started to accrue on the post-marketing occurrence of rare and unpredictable non-bleeding events with direct oral anticoagulants (DOACs), including liver injury and skin reactions, which has also raised debate on whether or not DOACs can be considered a homogeneous pharmacological class, especially from a safety perspective [1]. Cases of interstitial lung disease (ILD) have also been recently described, especially in Japanese subjects [2, 3], although no observational studies have comprehensively investigated post-marketing evidence of pulmonary toxicity with DOACs.

Therefore, we performed a disproportionality analysis in the Food and Drug Administration (FDA) Adverse Event Reporting System (FAERS) to investigate the reporting of ILD with DOACs over the period 2004-2019. The analyses were implemented on a de-duplicated dataset, by calculating the reporting odds ratio (ROR), deemed significant by the lower limit of the $95 \%$ confidence interval (CI) $>1$ with at least three cases. ILD was defined by the relevant preferred term in the Medical Dictionary for Regulatory Activity $\left(\right.$ MedDRA $\left.{ }^{\circledR}\right)$ terminology. Amiodarone, known to be associated with ILD, was used as a positive control, and warfarin was also analyzed as a reference. The main analysis was performed without pre-specified restriction criteria, i.e., considering all reports and comparing DOACs versus other drugs recorded in FAERS.

Emanuel Raschi

emanuel.raschi@unibo.it

1 Pharmacology Unit, Department of Medical and Surgical Sciences, Alma Mater Studiorum - University of Bologna, Via Irnerio 48, 40126 Bologna, Italy

2 Cardiology Unit, Department of Experimental, Diagnostic and Specialty Medicine, Alma Mater Studiorum - University of Bologna, Bologna, Italy
Sensitivity analyses were planned a priori to test the robustness of findings and assess the impact of potential confounders. Thus, we excluded reports recording (1) bleeding (apart from reports with co-reporting of ILD) using the Standardized MedDRA Query (SMQ) "Haemorrhage", since the expected high reporting of bleeding with DOACs can mask ILD (the so-called event-related competition bias); (2) amiodarone, expected to be frequently co-prescribed with DOACs in patients with atrial fibrillation (the so-called co-prescription bias); (3) drugs known to be strongly associated with ILD [4], which may act as a confounding factor and also mask identification of ILD with DOACs (the socalled drug-related competition bias), including anticancer and anti-rheumatic drugs; (4) antineoplastic and immunomodulating drugs (based on the Anatomical Therapeutic Chemical Classification System [ATC] code L), potentially acting as confounders since oncology patients receiving DOACs may develop ILD due to anticancer therapy or as neoplasia-related sequela (e.g., radiotherapy and/or pneumonitis); and (5) Japan as the reporter country, where high reporting of ILD is a key feature [5]. Additional analyses were undertaken, restricting them to suspect reports (i.e., primary/secondary suspect) and comparing DOACs versus other antithrombotic drugs. Time to onset (expressed in days, with interquartile range [IQR]) was also calculated (the difference between the start of therapy and the date the event occurred); for this purpose, only suspect cases where ILD was the single event recorded were used.

Increased reporting of ILD emerged with DOACs as a class across all analyses (962 cases with DOACs out of a total 24,720 cases); $60 \%$ were submitted by Asia (ILD accounted for $3.42 \%$ of Japanese reports, as compared to 0.12\% among European/North American reports), 34\% were cases in females, $87 \%$ were in individuals aged $>65$ years; and hospitalization occurred in $60 \%$ of cases. Death was calculated for reports where only ILD was recorded; it was $17 \%$ among all anticoagulants (18\% for anti-Xa agents) and ranged from $15 \%$ (apixaban) to $23 \%$ (edoxaban). "Atrial 


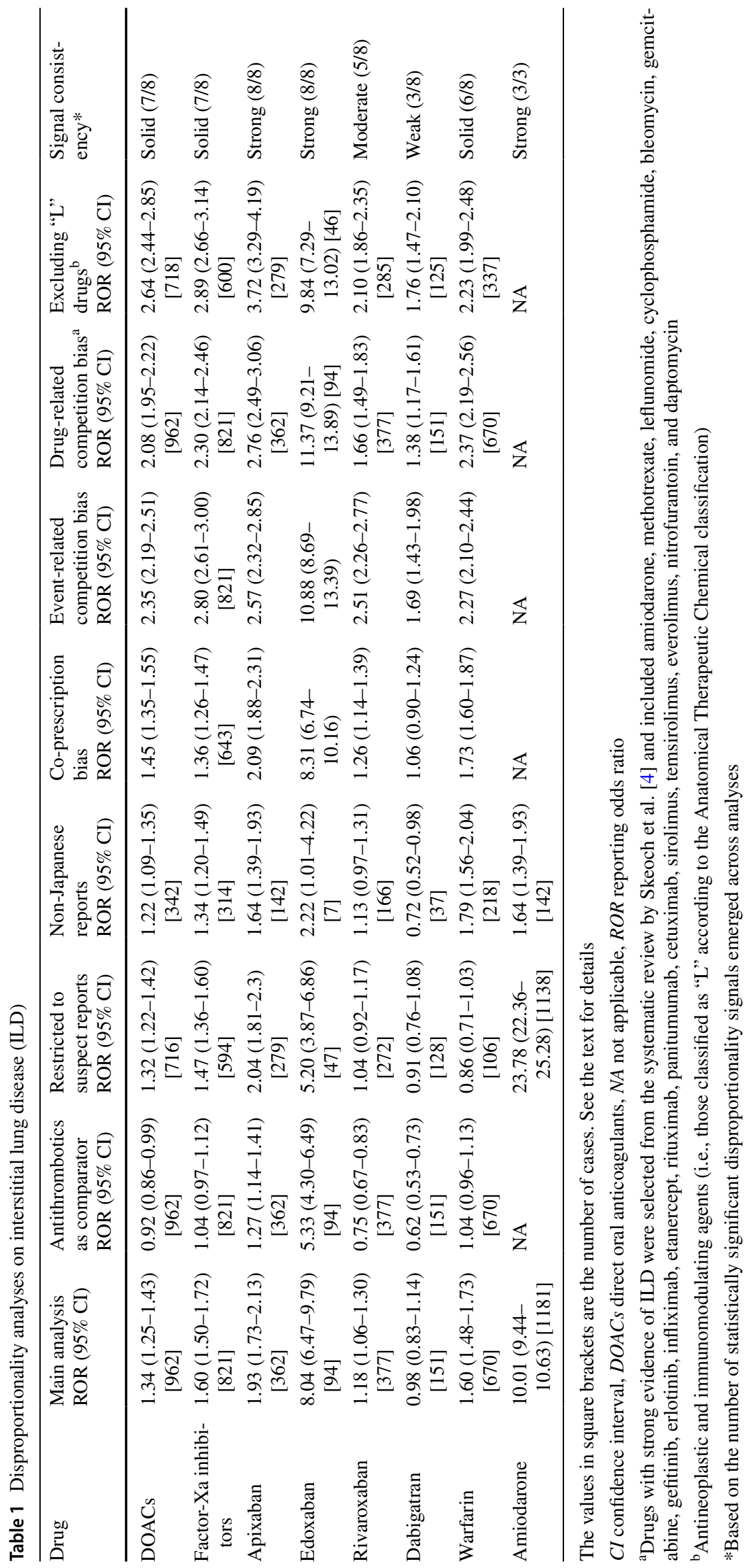


fibrillation" was the most frequently recorded therapeutic indication for dabigatran, rivaroxaban, and edoxaban $(68 \%$, $37 \%$, and $30 \%$, respectively), whereas "cerebrovascular accident prophylaxis" was found in $42 \%$ of apixaban cases. Methotrexate (2.9\% of ILD cases), rituximab (2.5\%), cyclophosphamide (1.6\%), and etanercept $(1.3 \%)$ were the most frequently co-reported drugs with known ILD risk.

We observed differences in terms of disproportionality signals (Table 1); apixaban and edoxaban consistently emerged with higher-than-expected reporting of ILD, with strongest disproportionality for edoxaban in the main analysis (ROR 8.04; 95\% CI 6.47-9.79), confirmed by sensitivity analyses. Conversely, dabigatran mainly resulted in non-significant disproportionality, and a signal emerged only when accounting for competition biases. Japanese reports had substantial impact on disproportionality (i.e., their removal caused a remarkable reduction in ROR estimates); only for rivaroxaban statistical significance disappeared. Median times to onset were 49 [IQR 15-176.5], 65 [40-182.5], and 147.5 days [21.5-550.5] for anti-Xa agents, dabigatran, and amiodarone, respectively (calculated for 163,47 , and 86 cases).

This hypothesis-generating study identified novel signals linking DOACs to ILD, especially for factor-Xa inhibitors, which showed anti-inflammatory and antifibrotic properties in pre-clinical models [6]. We put forward different hypotheses. First, we acknowledge that market penetration and Japan's regulatory system, including "important precautions" added to the rivaroxaban Japanese prescribing information on February 2014, may have contributed to the observed signals. Second, alveolar hemorrhage may occur (and manifest as ILD) with apixaban and rivaroxaban, which, respectively, have higher metabolic liability (drug interaction potential) and bleeding risk as compared to dabigatran [1]. Conversely, the lack of consistent disproportionality for dabigatran is in agreement with current evidence and may have a biological rationale: marked anti-inflammatory and antifibrotic effects were demonstrated in a bleomycin model of ILD [7], and preliminary clinical evidence in 15 patients with ILD and systemic sclerosis showed good tolerability of dabigatran without serious adverse events [8].

Further evidence is needed, especially to clarify the relationship between lung fibrosis and thrombosis. In fact, while a local and systemic prothrombotic state was demonstrated in idiopathic pulmonary fibrosis, clinical data on the efficacy of anticoagulants are conflicting (warfarin could even increase mortality), and chronic DOAC intake recently emerged as an independent predictor of decreased mortality in elderly patients with interstitial pneumonia associated with coronavirus disease 2019 (COVID-19), where thromboinflammation is likely to exist [9]. The latency of ILD occurrence, apparently rapid in terms of median values (with large variability), is in line with previous case reports [2, 3]; this suggests variability in patients' toxic response, underlines the importance of exploring the mechanistic basis, and raises the hypothesis that hypersensitivity pneumonitis, a form of ILD, cannot be excluded. Moreover, the possible modifying effect of ethnicity (beyond previous considerations) needs further assessment.

Considering the recognized limitations of pharmacovigilance (inability to infer causality and risk ranking, and lack of a denominator and clinical details, including radiological and non-imaging data and smoking status) and the aforementioned limbo surrounding DOACs' impact on ILD, large well designed observational studies and global real-world prospective registries, such as the Edoxaban Treatment in routiNe clinical prActice (ETNA) registry, are warranted to assess the relationship between DOACs (particularly factorXa inhibitors) and ILD, and elucidate the role of host-related risk factors, including clinical setting (atrial fibrillation versus venous thrombosis and pulmonary embolism), ethnicity, and genetics.

\section{Declarations}

Funding ER, ID, and EP are supported by institutional research funds (Ricerca Fondamentale Orientata). The study was not funded in whole or in part by any research grant or funding body.

Conflict of interest ID reports minor speaker fees from Bayer, Boehringer Ingelheim, Daiichi Sankyo, and Pfizer, outside the submitted work. All other authors have no conflicts of interest that are directly relevant to the content of this article.

Ethics approval Not applicable.

Consent to participate Not applicable.

Consent for publication Not applicable.

Availability of data and material Data supporting the findings of this study were derived from the following resource, available in the public domain: https://www.fis.fda.gov/extensions/FPD-QDE-FAERS/FPDQDE-FAERS.html.

Code availability Codes for the analyses can be made available by the authors upon reasonable request.

Author contributions ER made substantial contributions to study conception and design. MF made substantial contributions to the acquisition and analysis of data. ER, MF, ID, and EP made substantial contributions to the study design and interpretation of data. ER drafted the manuscript. All authors revised the manuscript critically for important intellectual content and approved the final version.

\section{References}

1. Raschi E, Bianchin M, Gatti M, Squizzato A, De Ponti F. Comparative effectiveness and safety of direct oral anticoagulants: overview of systematic reviews. Drug Saf. 2019;42(12):1409-22. 
2. Tomari S, Homma K, Noguchi T, et al. Development of interstitial lung disease after initiation of apixaban anticoagulation therapy. J Stroke Cerebrovasc Dis. 2016;25(7):1767-9.

3. Yanagihara T, Yamamoto N, Kotetsu Y, et al. Interstitial pneumonia caused by dabigatran. Respir Med Case Rep. 2017;23:10-2.

4. Skeoch S, Weatherley N, Swift AJ, et al. Drug-induced interstitial lung disease: a systematic review. J Clin Med. 2018;7(10):356.

5. Iwasa E, Fujiyoshi Y, Kubota Y, et al. Interstitial lung disease as an adverse drug reaction in Japan: exploration of regulatory actions as a basis for high reporting [published online ahead of print, 2020 Jul 2]. Drug Saf. 2020. https://doi.org/10.1007/s4026 4-020-00968-7.

6. Bukowska A, Schild L, Bornfleth P, et al. Activated clotting factor $\mathrm{X}$ mediates mitochondrial alterations and inflammatory responses via protease-activated receptor signaling in alveolar epithelial cells. Eur J Pharmacol. 2020;869:172875. https://doi. org/10.1016/j.ejphar.2019.172875.

7. Bogatkevich GS, Ludwicka-Bradley A, Nietert PJ, Akter T, van Ryn J, Silver RM. Antiinflammatory and antifibrotic effects of the oral direct thrombin inhibitor dabigatran etexilate in a murine model of interstitial lung disease. Arthritis Rheum. 2011;63(5):1416-25.

8. Silver RM, Wilson DA, Akter T, et al. Safety and tolerability of thrombin inhibition in scleroderma-associated interstitial lung disease. ACR Open Rheumatol. 2019;1(7):403-11.

9. Rossi R, Coppi F, Talarico M, Boriani G. Protective role of chronic treatment with direct oral anticoagulants in elderly patients affected by interstitial pneumonia in COVID-19 era. Eur J Intern Med. 2020;77:158-60. 